\title{
VARIABILIDAD TEMPORAL DE LA CONCENTRACIÓN DIARIA DE PRECIPITACIÓN EN LA ARGENTINA
}

\author{
María Paula Llano \\ Departamento de Ciencias de la Atmósfera y los Océanos, FCEyN, UBA. CONICET
}

(Manuscrito recibido el 1 de julio de 2020, en su versión final el 30 de agosto de 2020)

\section{RESUMEN}

En el presente estudio se analiza el comportamiento espacial y temporal de las precipitaciones mediante el índice de concentración diaria de precipitación con una resolución de 1, 5 y 10 milímetros para 24 estaciones meteorológicas en el período 1961-2018. El índice de concentración calculado con una resolución de $1 \mathrm{~mm}$ toma valores entre 0,58 y 0,67 , sin un patrón definido de comportamiento espacial. Con resolución de $5 \mathrm{~mm}$ y $10 \mathrm{~mm}$, los valores del índice disminuyen y a nivel espacial se hace notorio un gradiente norte - sur. En cuanto a la evolución de los valores de CI, la cantidad de estaciones con tendencias significativas negativas es similar a las positivas, mientras que en magnitud, son mayores en promedio las positivas. Este aumento en la concentración de la precipitación puede llegar a ser un riesgo dependiendo de la evolución de la lluvia asociada.

Palabras clave: concentración, precipitación diaria, tendencia.

\section{TEMPORAL VARIABILITY OF DAILY CONCENTRATION OF PRECIPITATION IN ARGENTINA}

\begin{abstract}
In the present study, the spatial and temporal behaviour of daily rainfall is analyzed using the daily rainfall concentration index with a resolution of 1,5 and 10 millimetres for 24 meteorological stations in the period 1961-2018. The index calculated with a resolution of $1 \mathrm{~mm}$ takes values between 0.58 and 0.67 , without a defined pattern of spatial behaviour. The CI $(5$ and $10 \mathrm{~mm})$ decrease and a spatial north-south gradient become noticeable. Analyzing the trends, the number of stations with significant negative trends is similar to positive ones, however, in magnitude, positive ones are higher on average. This increase in the concentration of precipitation could become a risk depending on the evolution of the associated precipitation.

Keywords: concentration, daily precipitation, trend
\end{abstract}




\section{INTRODUCCIÓN}

La importancia de estudiar las precipitaciones diarias radica en los problemas hidrológicos causados por las altas intensidades y la distribución desigual de las lluvias diarias a lo largo del territorio argentino. La ocurrencia de estos días particularmente lluviosos puede determinar si un año va a ser catalogado como seco o lluvioso. Las lluvias torrenciales pueden ocasionar catástrofes naturales como son las inundaciones, la erosión del suelo o el anegamiento de calles en las ciudades. La concentración de la precipitación puede calcularse mediante diferentes métodos. Uno de ellos se centra en la relación entre la acumulación de la variable y su frecuencia acumulativa. Esta función se conoce como curva de concentración o curva de Lorenz, y el área bajo esa curva es la base para el cálculo del índice de concentración de precipitación diaria CI (Martín-Vide, 2004). Este índice ha sido empleado en varias regiones del mundo: el CI fue calculado en varias regiones de Italia con diferentes enfoques y resultados (Coscarelli y Caloiero, 2012; Caloiero et al., 2019). En Nueva Zelanda dada la naturaleza de la isla los valores de CI presentan un amplio rango de variación (Caloiero, 2014). Analizando el comportamiento con el tiempo, en España la mayor parte del territorio presenta tendencia positiva en el CI en un amplio registro temporal (Serrano Notivoli et al., 2018), mientras que en el norte de China se observaron tendencias negativas en los últimos 50 años (Wang et al., 2019).

En el continente americano se puede mencionar el estudio hecho en Puerto Rico, donde se observa una gran variabilidad del CI dada la diferente topografía del país (Velez et al., 2019). En México, Núñez-González (2020) calcula el CI y realiza una comparación con otros países del mundo. En el noreste de Brasil el CI fue analizado por Fernandes Teixeira et al. (2014). En la zona centro-sur de Chile se analizan las tendencias del CI, encontrando resultados diferentes en el centro y en el sur (Sarricolea et al., 2019). Un estudio similar realizan Menseguer Ruiz et al. (2019) estudiando el comportamiento temporal del índice en la región norte de Chile. En los Andes de Perú el estudio de las tendencias de CI mostró que las mismas son variadas debido a la geografía de la región (Zubieta et al., 2017). En Argentina, Llano (2018) calculó el índice de concentración de precipitación diaria para 66 estaciones en el período 1991-2014 y realizó una compasión con otras regiones del mundo. En el presente trabajo se extiende el período de estudio de la concentración de la precipitación y se analiza la misma en base a su variabilidad espacial y temporal, como así también su relación con diferentes factores geográficos y climáticos.

\section{DATOS Y METODOLOGIAS}

Para la realización de la presente investigación se emplearon datos diarios de 24 estaciones meteorológicas para el período 1961-2018, las mismas fueron suministradas por el Servicio Meteorológico Nacional (SMN). El período de 58 años permite obtener resultados confiables de las metodologías aplicadas. En la Tabla I se detalla su ubicación geográfica, la altura (m) y la precipitación acumulada anual media $(\mathrm{mm})$, la cantidad promedio de días con precipitación al año y el coeficiente de variación (CV) que mide la relación entre el desvío estándar y el valor medio de la precipitación acumulada anual.

Es sabido que existen muchos días con precipitación escasa (inferior a $1 \mathrm{~mm}$ ) y pocos días con precipitaciones altas, por tal motivo la distribución de frecuencias de las precipitaciones diarias es posible ajustarla mediante una curva exponencial negativa (Brooks y Carruthers, 1953). El índice de concentración diaria de precipitación (CI) (Martin-Vide, 2004) es una herramienta que permite determinar la contribución relativa de los días de lluvia frente a la cantidad total de lluvia.

Para comenzar con el cálculo del CI es necesario clasificar a las precipitaciones diarias en intervalos de $1 \mathrm{~mm}$, comenzando por $(0-1]$, seguido por $(1-2],(2-3]$, etc. en orden creciente hasta alcanzar el máximo de los acumulados 


\begin{tabular}{|l|c|c|c|c|c|c|}
\hline \multicolumn{1}{|c|}{ Estaciones } & Longitud & Latitud & Altitud $(\mathrm{msnm})$ & $\begin{array}{c}\text { PP acu anual } \\
\text { media }(\mathrm{mm})\end{array}$ & $\begin{array}{c}N^{\circ} \text { de días con pp } \\
\text { por año medio }\end{array}$ & $\begin{array}{c}\text { Voeficiente de } \\
\text { Variación }\end{array}$ \\
\hline Salta & $-65,29$ & $-24,51$ & 1221 & 747,6 & 92,8 & 20,5 \\
\hline Las Lomitas & $-62,54$ & $-24,1$ & 130 & 910,2 & 81,1 & 22,0 \\
\hline Iguazú & $-54,28$ & $-25,44$ & 270 & 1840,6 & 104,2 & 26,8 \\
\hline Corrientes & $-58,46$ & $-27,27$ & 62 & 1411,1 & 96,4 & 25,7 \\
\hline Posadas & $-55,58$ & $-27,22$ & 125 & 1792,7 & 111,5 & 22,7 \\
\hline La Rioja & $-66,49$ & $-29,23$ & 429 & 386,0 & 46,4 & 31,5 \\
\hline Ceres & $-61,57$ & $-29,53$ & 88 & 930,9 & 83,8 & 22,9 \\
\hline Paso de los Libres & $-57,09$ & $-29,41$ & 70 & 1494,9 & 90,6 & 27,0 \\
\hline Sauce Viejo & $-60,49$ & $-31,42$ & 18 & 1022,5 & 76,2 & 22,1 \\
\hline Paraná & $-60,29$ & $-31,47$ & 78 & 1110,7 & 84,4 & 20,0 \\
\hline Monte Caseros & $-57,39$ & $-30,16$ & 54 & 1469,1 & 96,3 & 20,9 \\
\hline Mendoza & $-68,47$ & $-32,5$ & 704 & 213,4 & 40,8 & 40,9 \\
\hline Villa Reynolds & $-65,23$ & $-33,44$ & 486 & 707,2 & 76,7 & 24,5 \\
\hline Rosario & $-60,47$ & $-32,55$ & 25 & 1029,1 & 83,7 & 20,6 \\
\hline Gualeguaychú & $-58,37$ & -33 & 23 & 1140,2 & 89,7 & 26,6 \\
\hline Laboulaye & $-63,22$ & $-34,08$ & 137 & 889,1 & 85,8 & 21,7 \\
\hline Pehuajó & $-61,54$ & $-35,52$ & 87 & 935,2 & 81,6 & 21,9 \\
\hline Junín & $-60,55$ & $-34,33$ & 81 & 1049,1 & 84,7 & 22,1 \\
\hline Ezeiza & $-58,32$ & $-34,49$ & 20 & 1002,7 & 90,5 & 20,2 \\
\hline Santa Rosa & $-64,16$ & $-36,34$ & 191 & 723,6 & 74,2 & 27,8 \\
\hline Neuquén & $-68,08$ & $-38,57$ & 271 & 198,8 & 46,3 & 47,3 \\
\hline Bahía Blanca & $-62,1$ & $-38,44$ & 83 & 642,3 & 77,8 & 23,1 \\
\hline Esquel & $-71,09$ & $-42,56$ & 797 & 478,3 & 79,9 & 25,0 \\
\hline Río Gallegos & $-69,17$ & $-51,37$ & 19 & 255,2 & 91,4 & 25,3 \\
\hline
\end{tabular}

Tabla I: Ubicación geográfica de las 24 estaciones (longitud, latitud y altitud), junto con parámetros medios: precipitación acumulada anual, número de días con precipitación y coeficiente de variación..

diarios que se produjo en el período de análisis. En la Tabla II se muestra a modo de ejemplo el cálculo realizado para la estación Ezeiza. En la primera columna están los intervalos mencionados y en la segunda se encuentra el valor medio de cada uno de ellos. En la tercera columna se consignan las frecuencias absolutas de cada intervalo $\left(n_{i}\right)$. En la cuarta columna se presenta la frecuencia absoluta acumulada $\Sigma \mathrm{n}_{i}$ (el último valor corresponde a la cantidad total de días con precipitación). En Ezeiza en los 58 años de registro hubo 5249 días con precipitación. En la quinta columna se encuentran los valores de $P_{i}$, que corresponden a la multiplicación entre la marca de clase $\left(2^{\circ}\right.$ columna) y la frecuencia ( $3^{\circ}$ columna). En la sexta columna se hallan los valores acumulados de la columna anterior $\Sigma \mathrm{P}_{i}$. Finalmente en la séptima y octava columna están los porcentajes de los valores de las columnas cuarta y sexta con respecto a los últimos valores de las mismas: $\Sigma \mathrm{n}_{i}$ (\%) y $\Sigma \mathrm{P}_{i}(\%)$. La representación gráfica de estas dos últimas columnas da lugar a la curva marcadamente exponencial que se denomina curva de concentración o curva de Lorenz.

A su vez, se calcula el mismo índice pero con intervalos de $5 \mathrm{~mm}$ y $10 \mathrm{~mm}$ (Benhamrouche y Martin-Vide, 2018), lo que permite trabajar con estaciones en donde el rigor de los registros es menor (no fueron precisas las mediciones o no se registraron diariamente). El índice de concentración cuantifica el área entre la curva exponencial y la recta de equidistribución. La curva exponencial tiene la forma de $Y=a X \exp (b X), \mathrm{y}$ las constantes a $\mathrm{y} \mathrm{b}$ se obtienen por el método de cuadrados mínimos a partir de $X_{i}=\Sigma \mathrm{n}_{i}(\%)$ e $Y_{i}=\Sigma \mathrm{P}_{i}(\%)$ : 


$$
\begin{gathered}
\ln a=\frac{\sum X_{i}^{2} \sum \ln Y_{i}+\sum X_{i} \sum X_{i} \ln X_{i}-\sum X_{i}^{2} \sum \ln X_{i}-\sum X_{i} \sum X_{i} \ln Y_{i}}{N \sum X_{i}^{2}-\left(\sum X_{i}\right)^{2}} \\
b=\frac{N \sum X_{i} \ln Y_{i}+\sum X_{i} \sum \ln X_{i}-N \sum X_{i} \ln X_{i}-\sum X_{i} \sum \ln Y_{i}}{N \sum X_{i}^{2}-\left(\sum X_{i}\right)^{2}}
\end{gathered}
$$

\begin{tabular}{|c|c|c|c|c|c|c|c|}
\hline Intervalo & Valor medio & $\mathbf{n}_{\mathbf{i}}$ & $\boldsymbol{\Sigma} \mathbf{n}_{\mathbf{i}}$ & $\mathbf{P}_{\mathbf{i}}$ & $\boldsymbol{\Sigma} \mathbf{P}_{\mathbf{i}}$ & $\boldsymbol{\Sigma} \mathbf{n}_{\mathbf{i}}(\%)=\mathbf{X}$ & $\boldsymbol{\Sigma} \mathbf{P}_{\mathbf{i}}(\%)=\mathbf{Y}$ \\
\hline $0,1-1$ & 0,5 & 1296 & 1296 & 648 & 648 & 24,69 & 1,13 \\
\hline $1,1-2$ & 1,5 & 451 & 1747 & 676,5 & 1324,5 & 33,28 & 2,30 \\
\hline $2,1-3$ & 2,5 & 351 & 2098 & 877,5 & 2202 & 39,97 & 3,82 \\
\hline $3,1-4$ & 3,5 & 290 & 2388 & 1015 & 3217 & 45,49 & 5,59 \\
\hline $4,1-5$ & 4,5 & 265 & 2653 & 1192,5 & 4409,5 & 50,54 & 7,66 \\
\hline $5,1-6$ & 5,5 & 192 & 2845 & 1056 & 5465,5 & 54,20 & 9,49 \\
\hline $6,1-7$ & 6,5 & 204 & 3049 & 1326 & 6791,5 & 58,09 & 11,79 \\
\hline $7,1-8$ & 7,5 & 168 & 3217 & 1260 & 8051,5 & 61,29 & 13,98 \\
\hline $8,1-9$ & 8,5 & 153 & 3370 & 1300,5 & 9352 & 64,20 & 16,24 \\
\hline $9,1-10$ & 9,5 & 138 & 3508 & 1311 & 10663 & 66,83 & 18,52 \\
\hline $10,1-11$ & 10,5 & 91 & 3599 & 955,5 & 11618,5 & 68,57 & 20,18 \\
\hline $11,1-12$ & 11,5 & 111 & 3710 & 1276,5 & 12895 & 70,68 & 22,39 \\
\hline $12,1-13$ & 12,5 & 94 & 3804 & 1175 & 14070 & 72,47 & 24,43 \\
\hline $13,1-14$ & 13,5 & 86 & 3890 & 1161 & 15231 & 74,11 & 26,45 \\
\hline $14,1-15$ & 14,5 & 75 & 3965 & 1087,5 & 16318,5 & 75,54 & 28,34 \\
\hline $15,1-16$ & 15,5 & 90 & 4055 & 1395 & 17713,5 & 77,25 & 30,76 \\
\hline $16,1-17$ & 16,5 & 71 & 4126 & 1171,5 & 18885 & 78,61 & 32,80 \\
\hline $17,1-18$ & 17,5 & 67 & 4193 & 1172,5 & 20057,5 & 79,88 & 34,83 \\
\hline $18,1-19$ & 18,5 & 57 & 4250 & 1054,5 & 21112 & 80,97 & 36,66 \\
\hline $19,1-20$ & 19,5 & 52 & 4302 & 1014 & 22126 & 81,96 & 38,42 \\
\hline $20,1-21$ & 20,5 & 63 & 4365 & 1291,5 & 23417,5 & 83,16 & 40,67 \\
\hline$\ldots$ & & & & & & & \\
\hline $124,1-125$ & 124,5 & 1 & 5246 & 124,5 & 57199 & 99,94 & 99,33 \\
\hline $125,1-126$ & 125,5 & 1 & 5247 & 125,5 & 57324,5 & 99,96 & 99,55 \\
\hline $126,1-127$ & 126,5 & 0 & 5247 & 0 & 57324,5 & 99,96 & 99,55 \\
\hline $127,1-128$ & 127,5 & 1 & 5248 & 127,5 & 57452 & 99,98 & 99,77 \\
\hline $128,1-129$ & 128,5 & 0 & 5248 & 0 & 57452 & 99,98 & 99,77 \\
\hline $129,1-130$ & 129,5 & 0 & 5248 & 0 & 57452 & 99,98 & 99,77 \\
\hline $130,1-131$ & 130,5 & 1 & 5249 & 130,5 & 57582,5 & 100,00 & 100,00 \\
\hline & & & & & & & \\
\hline
\end{tabular}

Tabla II: Distribución de frecuencias en intervalos de $1 \mathrm{~mm}$, frecuencia acumulada $\mathrm{X}$ y su correspondiente porcentaje del total de precipitación $\mathrm{Y}$, para la estación Ezeiza (1961-2018).

Con ambas constantes se puede calcular el área bajo la curva exponencial, mediante la siguiente integral entre 0 y 100.

$$
A=\left[\frac{a}{b} e^{b x}\left(x-\frac{1}{b}\right)\right]_{0}^{100}
$$

El área S' que está comprendida entre la curva y la línea de distribución equitativa y $X=100$ es la diferencia entre 5000 y el valor de la ecuación anterior. Por lo tanto el índice de concentración de precipitación diaria se define como:

$$
C I=S^{\prime} / 5000
$$

En la Figura 1, se observa la curva de concentración para la estación Ezeiza para los tres cálculos CI 1mm, CI 5mm y CI $10 \mathrm{~mm}$. Ezeiza tiene un valor de CI igual a 0,63,0,58 y 0,54 respectivamente, lo que muestra las diferencias al tomar los tres intervalos. Este tipo de gráfica permite ver la relación entre el porcentaje de días con precipitación versus el porcentaje de precipitación. Dado que el CI brinda una medida del área, un índice elevado indica que la distribución de la precipitación diaria no se comporta de manera regular (alejado de la recta de equidistribución).

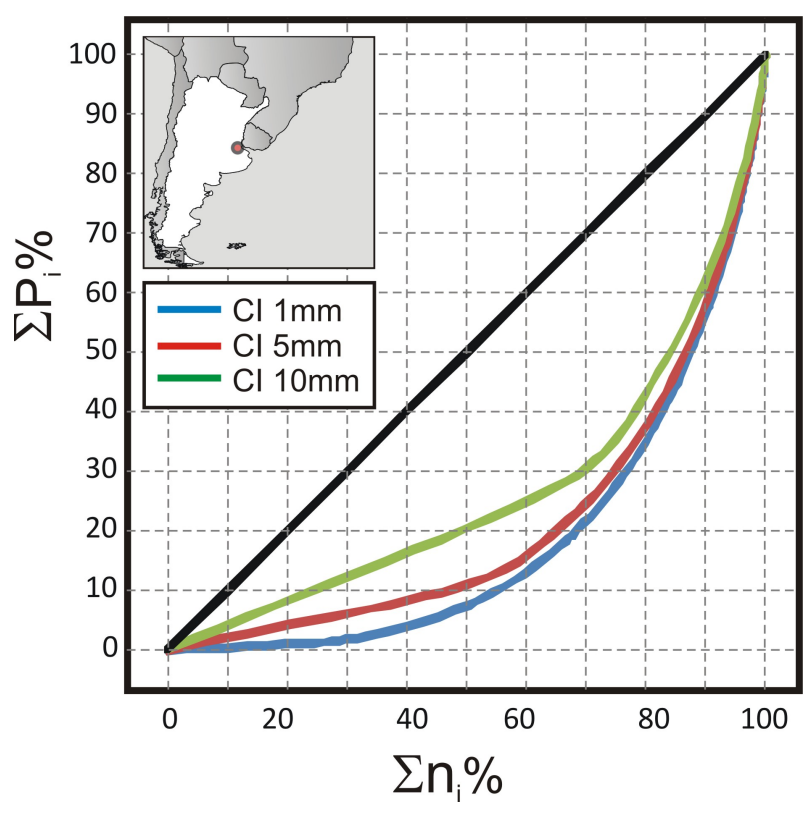

Figura 1: Curvas de concentración o curvas de Lorenz para la estación Ezeiza para las tres categorías de CI.

En el presente trabajo se estudia la relación lineal entre los índices concentración y diferentes parámetros geográficos de las estaciones (longitud, latitud, altitud), como así también con parámetros medios de la precipitación (precipitación acumulada anual media, cantidad de días con precipitación al año media, coeficiente de variación). Para finalizar 
se estudia la tendencia y su significancia por medio del test no paramétrico de Mann Kendall (Mann 1945, Kendall 1962) y la estimación no paramétrica de Sen (1968) se empleará para conocer la pendiente (siendo la pendiente el valor de la mediana entre todas las pendientes dadas entre pares de datos consecutivos), utilizando el paquete estadístico "trend" para R (Pohlert, 2018).

\section{RESULTADOS}

En la Tabla III se presentan los valores de CI para las tres clases de $1 \mathrm{~mm}, 5 \mathrm{~mm}$ y $10 \mathrm{~mm}$ (CI1 - CI5 - CI10) en conjunto con el porcentaje de la precipitación aportada por el 25\% (Pi25\%) y $10 \%$ (Pi10\%) de los días más lluviosos en las estaciones de referencia para el período completo 1961-2018.

Los valores de CI1 se encuentran entre 0,58 y 0,67. El máximo se produce en dos localidades Las Lomitas y Ceres, donde el $25 \%$ de los días más lluviosos están aportando 77,9\% y 78,5\% de la precipitación total, respectivamente. Si se analiza el $10 \%$ de los días más lluviosos en estas estaciones se estaría aportando un $46,3 \%$ y 46,9\% de la precipitación; esto estaría indicando que en las localidades con un CI cercano a 0,67 aproximadamente $50 \%$ de la precipitación se concentra en el $10 \%$ de los días considerados más lluviosos y el otro $50 \%$ de la precipitación se reparte en el $90 \%$ de los días restantes. En el otro extremo, las localidades con menor índice de concentración son Esquel $(0,58)$, Iguazú $(0,59)$, Río Gallegos $(0,60)$ y Paso de los Libres $(0,60)$, con estos valores de CI el $25 \%$ de los días más lluviosos representan un menor porcentaje de la precipitación total entre un 70 y $72 \%$. El $10 \%$ de los días más lluviosos representan aproximadamente un $40 \%$ de la precipitación total. En promedio en la Argentina el valor de CI en la categoría de $1 \mathrm{~mm}$ es de 0,63. Lo que estaría indicando que el $25 \%$ de los días más lluviosos aportan un $74 \%$ de la precipitación total y el $10 \%$ más lluvioso aporta un $42 \%$.
El CI puede tomar valores entre 0 y 1 . En la categoría de $1 \mathrm{~mm}$ los valores clasificarse en 5 categorías: muy baja concentración $(0-0,2)$, baja concentración $(0,2-0,4)$, concentración moderada $(0,4-0,6)$, alta concentración $(0,6-0,8)$ y muy alta concentración (0,8-1) (Núñez-González, 2020). La alta concentración de precipitación está vinculada a los procesos físicos rápidos, como la convección. Mientras que, la baja concentración de lluvia puede interpretarse como consecuencia de patrones regulares, como el paso sucesivo de sistemas de bajas presiones o tormentas (Monjo y Martín-Vide, 2016). Al tomar un intervalo de clase de 5 milímetros, los valores de CI cubren un rango: $0,50-0,63$. Nuevamente las dos estaciones con valores más altos de CI son Ceres y Las Lomitas, analizando el $25 \%$ de los días más lluviosos los resultados de las curvas teóricas muestran que los mismos aportan un 72,9 y 73,7\% de la precipitación total respectivamente, y el $10 \%$ de los días más lluviosos aporta un $42 \%$. Las localidades con valores más bajos presentan un comportamiento diferente al visto para CI 1, Río Gallegos se aparta del resto con un valor de 0,50, y es seguida por Esquel con 0,54 y con 0,56 se encuentra a Iguazú y Villa Reynolds. El $25 \%$ de los días más lluviosos en Río Gallegos aportan un $59 \%$ de la precipitación y al restringir a un $10 \%$ el aporte se reduce a un $30 \%$. En las otras localidades mencionadas estos valores aumentan. Cuando el intervalo asciende a 10 milímetros el rango de variación del índice es de 13 centésimas al igual que el caso anterior, pero los valores abarcan desde 0,46 a 0,59 . Las estaciones de Ceres y Las Lomitas vuelven a presentar el valor máximo $(0,59)$, con este CI el $25 \%$ de los días más lluviosos están aportando alrededor de un $69 \%$ de la precipitación, mientras que un $38 \%$ de la precipitación se concentra en el $10 \%$ de los días más lluviosos. En el extremo opuesto del índice se encuentran la estación más austral, Río Gallegos con un CI de 0,46 , concentra un $27 \%$ de la precipitación en el $10 \%$ de los días más lluviosos y un $55 \%$ de la lluvia en el $25 \%$ de los días más lluviosos. En la Figura 2 se muestra la distribución espacial 


\begin{tabular}{|l|c|c|c|c|c|c|c|c|c|}
\hline Estaciones & $\mathbf{C I} \mathbf{1} \mathbf{m m}$ & $25 \% 1 \mathrm{~mm}$ & $10 \% 1 \mathrm{~mm}$ & $\mathbf{C I} \mathbf{5 m m}$ & $25 \% 5 \mathrm{~mm}$ & $10 \% 5 \mathrm{~mm}$ & $\mathbf{C I} \mathbf{1 0} \mathbf{~ m m}$ & $25 \% 10 \mathrm{~mm}$ & $10 \% 10 \mathrm{~mm}$ \\
\hline Salta & $\mathbf{0 , 6 6}$ & 76,5 & 45,7 & $\mathbf{0 , 6 1}$ & 70,5 & 40,3 & $\mathbf{0 , 5 6}$ & $\mathbf{6 5 , 9}$ & 36,6 \\
\hline Las Lomitas & $\mathbf{0 , 6 7}$ & 77,9 & 46,3 & $\mathbf{0 , 6 3}$ & 73,7 & 42,6 & $\mathbf{0 , 5 9}$ & 69,0 & 38,4 \\
\hline Iguazú & $\mathbf{0 , 5 9}$ & 72,0 & 39,6 & $\mathbf{0 , 5 6}$ & 68,1 & 37,6 & $\mathbf{0 , 5 3}$ & 64,3 & 34,7 \\
\hline Corrientes & $\mathbf{0 , 6 5}$ & 76,3 & 44,3 & $\mathbf{0 , 6 1}$ & 71,7 & 40,5 & $\mathbf{0 , 5 8}$ & 67,7 & 37,4 \\
\hline Posadas & $\mathbf{0 , 6 3}$ & 74,7 & 42,6 & $\mathbf{0 , 5 9}$ & 70,0 & 39,1 & $\mathbf{0 , 5 5}$ & 66,2 & 36,4 \\
\hline La Rioja & $\mathbf{0 , 6 3}$ & 73,9 & 42,8 & $\mathbf{0 , 5 8}$ & 68,1 & 38,3 & $\mathbf{0 , 5 3}$ & 63,0 & 34,2 \\
\hline Ceres & $\mathbf{0 , 6 7}$ & 78,5 & 46,9 & $\mathbf{0 , 6 3}$ & 72,9 & 42,0 & $\mathbf{0 , 5 9}$ & 68,6 & 38,4 \\
\hline Paso de los Libres & $\mathbf{0 , 6 0}$ & 72,7 & 40,5 & $\mathbf{0 , 5 7}$ & 68,6 & 38,0 & $\mathbf{0 , 5 4}$ & 64,7 & 34,9 \\
\hline Sauce Viejo & $\mathbf{0 , 6 2}$ & 73,5 & 41,5 & $\mathbf{0 , 5 8}$ & 68,9 & 38,2 & $\mathbf{0 , 5 5}$ & 65,1 & 35,5 \\
\hline Paraná & $\mathbf{0 , 6 4}$ & 75,4 & 43,6 & $\mathbf{0 , 6 0}$ & 70,6 & 39,8 & $\mathbf{0 , 5 7}$ & 66,6 & 36,5 \\
\hline Monte Caseros & $\mathbf{0 , 6 3}$ & 74,5 & 42,3 & $\mathbf{0 , 5 9}$ & 70,1 & 39,5 & $\mathbf{0 , 5 6}$ & 66,2 & 36,4 \\
\hline Mendoza & $\mathbf{0 , 6 4}$ & 75,1 & 44,4 & $\mathbf{0 , 5 8}$ & 67,5 & 37,7 & $\mathbf{0 , 5 3}$ & 62,2 & 33,4 \\
\hline Villa Reynolds & $\mathbf{0 , 6 1}$ & 72,8 & 41,4 & $\mathbf{0 , 5 6}$ & 66,9 & 37,3 & $\mathbf{0 , 5 3}$ & 62,7 & 34,0 \\
\hline Rosario & $\mathbf{0 , 6 4}$ & 75,1 & 43,3 & $\mathbf{0 , 6 0}$ & 70,3 & 39,7 & $\mathbf{0 , 5 6}$ & 65,8 & 36,3 \\
\hline Gualeguaychí & $\mathbf{0 , 6 4}$ & 75,8 & 43,9 & $\mathbf{0 , 6 0}$ & 70,7 & 40,0 & $\mathbf{0 , 5 6}$ & 66,5 & 36,7 \\
\hline Laboulaye & $\mathbf{0 , 6 5}$ & 76,2 & 44,1 & $\mathbf{0 , 6 0}$ & 70,1 & 39,0 & $\mathbf{0 , 5 5}$ & 64,9 & 35,1 \\
\hline Pehuajó & $\mathbf{0 , 6 3}$ & 74,7 & 43,1 & $\mathbf{0 , 5 9}$ & 69,4 & 38,8 & $\mathbf{0 , 5 5}$ & 65,1 & 35,4 \\
\hline Junín & $\mathbf{0 , 6 2}$ & 74,0 & 42,1 & $\mathbf{0 , 5 8}$ & 68,9 & 38,4 & $\mathbf{0 , 5 5}$ & 64,9 & 35,3 \\
\hline Ezeiza & $\mathbf{0 , 6 3}$ & 74,3 & 42,4 & $\mathbf{0 , 5 8}$ & 68,8 & 38,6 & $\mathbf{0 , 5 4}$ & 64,6 & 35,2 \\
\hline Santa Rosa & $\mathbf{0 , 6 2}$ & 74,1 & 42,5 & $\mathbf{0 , 5 8}$ & 68,2 & 37,7 & $\mathbf{0 , 5 4}$ & 63,8 & 34,5 \\
\hline Neuquén & $\mathbf{0 , 6 5}$ & 75,9 & 45,1 & $\mathbf{0 , 5 8}$ & 67,7 & 37,8 & $\mathbf{0 , 5 3}$ & 61,8 & 32,8 \\
\hline Bahía Blanca & $\mathbf{0 , 6 3}$ & 74,6 & 43,3 & $\mathbf{0 , 5 8}$ & 68,7 & 38,6 & $\mathbf{0 , 5 5}$ & 64,2 & 34,5 \\
\hline Esquel & $\mathbf{0 , 5 8}$ & 70,2 & 39,3 & $\mathbf{0 , 5 4}$ & 63,9 & 34,7 & $\mathbf{0 , 5 0}$ & 59,7 & 31,2 \\
\hline Río Gallegos & $\mathbf{0 , 6 0}$ & 70,3 & 39,3 & $\mathbf{0 , 5 0}$ & 59,4 & 30,6 & $\mathbf{0 , 4 6}$ & 55,2 & 27,7 \\
\hline
\end{tabular}

Tabla III: Valores de los índices de concentración para las 3 categorías de intervalo (CI 1mm, CI $5 \mathrm{~mm}$ y CI $10 \mathrm{~mm}$ ) con sus respectivos porcentajes de precipitación total que aportan el $25 \%$ y el $10 \%$ de los días más lluviosos.

de los índices calculados con intervalo de $1 \mathrm{~mm}$, $5 \mathrm{~mm}$ y $10 \mathrm{~mm}$. En el primer caso la distribución es aleatoria con diferentes centros de valores mayores o menores distribuidos a lo largo del todo el país, similar a lo previamente hallado por Llano (2018) con un mayor número de estaciones pero en menor período de tiempo. Los índices CI5 y CI10 muestran en mayor detalle el gradiente norte - sur que existe en el país. El sur del país exhibe los menores valores de CI dado el régimen de lluvias caracterizado por ser regular, mientras que el norte del país se caracteriza por tener un alto porcentaje de la precipitación concentrado en pocos días generalmente asociados a sistemas convectivos de mesoescala (Nesbitt et al., 2016). A medida que aumenta el intervalo de categorización de las precipitaciones, el índice es cada vez más homogéneo a nivel regional, se pierden las particularidades que una estación puede presentar en las lluvias diarias por lo tanto es más similar el valor del CI con estaciones cercanas. Es en la Patagonia donde más importante es el gradiente, Río Gallegos tiene un valor menor en casi 10 centésimas al hallado para Neuquén o Bahía Blanca.

A nivel país, el valor medio de los índices es: CI $1=0,63 ;$ CI $5=0,58$ y CI $10=0,54$. Se observa la relación inversa entre el valor del índice y la longitud de la clase. Al pasar de una longitud de $1 \mathrm{~mm}$ a $5 \mathrm{~mm}$, el valor del CI disminuye un $8 \%$ ( 5 centésimas); a su vez, el rango de valores (diferencia entre máximo y mínimo) que toma el CI aumenta (de 0,09 a 0,14). Entre los CI de $5 \mathrm{~mm}$ y $10 \mathrm{~mm}$, solo se observa una disminución del mismo en un $7 \%$ promedio país.

\subsection{Relaciones entre el CI y diferentes variables}

Con el objetivo de evaluar la correlación 

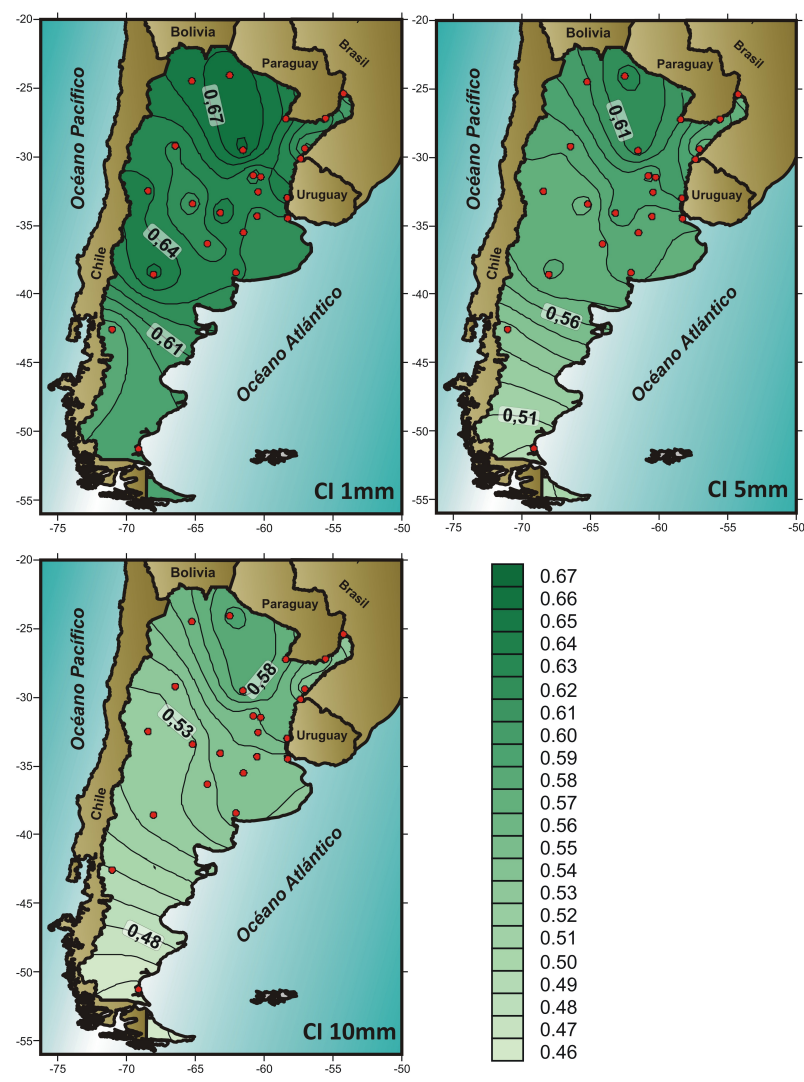

Figura 2: Isolíneas de CI para las 3 categorías (CI 1mm, CI5mm, CI10mm, derecha) para el período 1961-2018.

espacial entre el CI y diferentes parámetros, en esta Tabla IV se presentan los valores del coeficiente de correlación de Pearson entre el CI, calculado con los 3 intervalos y diferentes variables climáticas: precipitación anual acumulada media, cantidad de días con precipitación al año media, coeficiente de variación anual y tres variables geográficas: longitud, latitud y altitud de la estación. Estos valores están expresados en la Tabla I.

Las variables climáticas son las que presentan una menor relación con el CI, solo entre la precipitación acumulada y el CI 10 la relación es positiva y significativa. Entre las variables geográficas la latitud para los 3 CI muestra una relación positiva y significativa, hay que recordar que la latitud está expresada en valores negativos por lo que este resultado está indicando que a medida que nos desplazamos

\begin{tabular}{c|c|c|c|} 
& $\mathbf{C l} \mathbf{1} \mathbf{m m}$ & $\mathbf{C l} \mathbf{5} \mathbf{m m}$ & $\mathbf{C l} \mathbf{1 0} \mathbf{~ m m}$ \\
\hline pp_acu & $-0,09$ & 0,31 & $\mathbf{0 , 4 8}$ \\
\hline dias & $-0,19$ & 0,04 & 0,22 \\
\hline $\mathrm{CV}$ & 0,08 & $-0,18$ & $-0,33$ \\
\hline longitud & 0,04 & $\mathbf{0 , 4 1}$ & $\mathbf{0 , 5 5}$ \\
\hline latitud & $\mathbf{0 , 4 4}$ & $\mathbf{0 , 7 6}$ & $\mathbf{0 , 7 9}$ \\
\hline altitud & $-0,03$ & $-0,10$ & $-0,17$ \\
\hline
\end{tabular}

Tabla IV: Coeficiente de correlación de Pearson entre los 3 índices y la precipitación anual acumulada media, la cantidad de días con precipitación media, el coeficiente de variación, la longitud, la latitud y la altitud de las estaciones. En negrita los valores significativos al $5 \%$..

hacia el sur el CI disminuye. Este resultado está de acuerdo a lo visto en el apartado anterior. Con la longitud solo los índices CI 5 y CI 10 (cuya distribución es menos aleatoria) presentan correlación significativa y positiva, en esta oportunidad la relación está indicando que en el oeste del país los valores del índice son menores. Con la altitud no se encontró ningún tipo de relación significativa.

\subsection{Valores anuales del índice de concentración de precipitación}

Los valores de los índices fueron calculados en escala anual para cada uno de los 58 años. Con estas series se analiza la existencia de una tendencia por medio del test no paramétrico de Mann Kendall (MK) y la pendiente se la calcula por medio de la estimación Sen. Para el CI 1, desde el punto de vista de la distribución espacial de las tendencias, en la Figura 3 se puede observar como el centro - sur del país está dominado por valores positivos, lo que indica que con los años las lluvias están cada vez más concentradas, con la excepción de Neuquén y Bahía Blanca. En el sur del litoral y el norte de la provincia de Buenos Aires, las concentraciones son cada vez menores. En el noreste el comportamiento es diverso, no hay un claro patrón. Mientras que el noroeste las concentraciones están disminuyendo con el tiempo. 


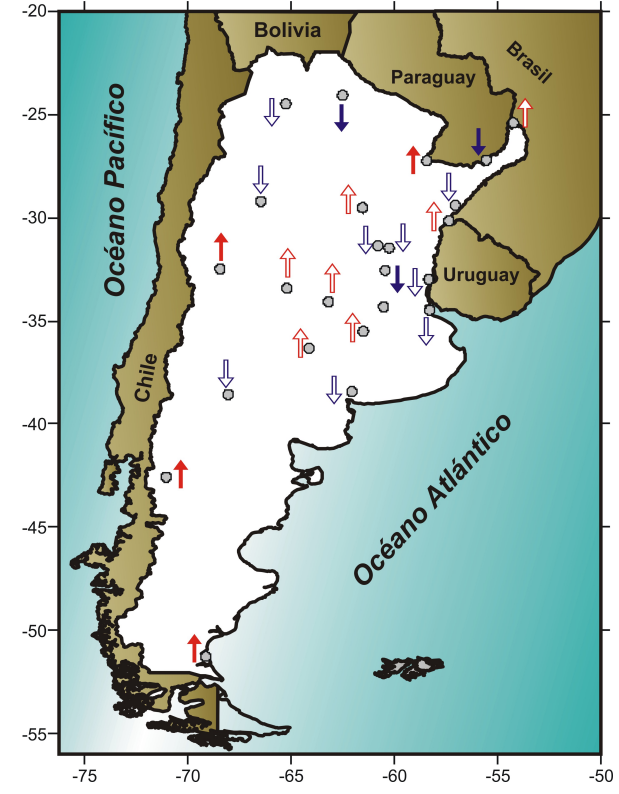

Figura 3: Tendencias para los valores anuales de CI 1mm según Mann Kendall (1961-2018). Flechas llenas significativas al $5 \%$, rojas (azules) positivas (negativas).

Como se pudo observar en la figura anterior, el test MK arrojó que ocho estaciones presentan una tendencia significativa, de las cuales la mitad son positivas y la mitad negativas. Para mayor detalle en la Figura 4 se presentan los valores de CI 1 anuales para estas ocho estaciones. Entre las tendencias negativas Las Lomitas se destaca por su pendiente presentando una disminución de CI de -0,0012 por año. Las otras 3 estaciones: Posadas, Rosario y Junín, tienen una disminución similar entre $-0,0004$ y -0,0006 por año. En el caso de las pendientes positivas, Corrientes, Mendoza, Esquel y Río Gallegos, presentan aumentos entre 0,0008 y 0,001 al año. Todas estas pendientes significativas son de menor magnitud a las vistas por Wang et al. (2019) en China, pero similares a varias de las estaciones de Cerdeña (Italia) estudiadas por Caloiero et al. (2019).

A nivel anual se puede observar el comportamiento disperso de este índice con el tiempo, presentando una gran variabilidad año a año, con diferencias promedios entre años consecutivos de entre un 5 y un $9 \%$ del valor del CI medio. Esta variabilidad interanual presenta valores máximos entre un 15 y un $20 \%$ del valor del CI, el caso más extremo con una diferencia entre años consecutivos de un $28 \%$ del valor del CI sucede en Mendoza entre los años 1996 y 1997. Tres estaciones con tendencia positiva tienen el mayor rango de valores, Mendoza presenta el mínimo de 0,48 en 1969 y el máximo en 0,75 en 1979, en Río Gallegos 0,48 en 1980 y 0,71 en 2018, finalmente en Esquel los extremos se producen en $1985(0,49)$ y $2015(0,69)$. Analizando el comportamiento de las tendencias se pueden mencionar algunos casos singulares como por ejemplo, Las Lomitas que en base a los bajos valores del CI de los últimos 10 años, la tendencia es significativa y negativa. En el otro extremo del país, Río Gallegos tiene una clara pendiente positiva con el paso del tiempo, la cual también se ve forzada por el valor de 0,71 del último año del registro, pero si se cambia el período de análisis (comenzando en la década del 80) la misma puede ser mucho mayor. Cuando se analiza la tendencia del índice CI 5, solo tres estaciones Las Lomitas, Corrientes y Río Gallegos exhiben disminución (la primera) y aumentos significativos (las 2 restantes). El índice CI 10 no presenta cambios significativos con el tiempo. Este comportamiento puede deberse, como ya se mencionó anteriormente, a que con intervalos de clase más grandes los eventos aislados de alta concentración de precipitación se ven mitigados y los años con CI extremos que podrían producir una tendencia significativa están suavizados. Por último, se estudian las tendencias de la precipitación anual acumulada mediante el test de MK. La mayoría de las estaciones exhibe un aumento de la precipitación, siendo significativas las tendencias en Las Lomitas, Iguazú, Posadas, Sauce Viejo, Paraná, Mendoza. Gualeguaychú, Junín y Rio Gallegos. Solo dos estaciones presentaron una disminución no significativa en la cantidad de precipitación anual: Bahía Blanca y Esquel. $\mathrm{Al}$ analizar el comportamiento conjunto en las estaciones donde las tendencias de CI 1 y de la precipitación acumulada son significativas, se hallan 2 tipos de comportamiento: aumento 

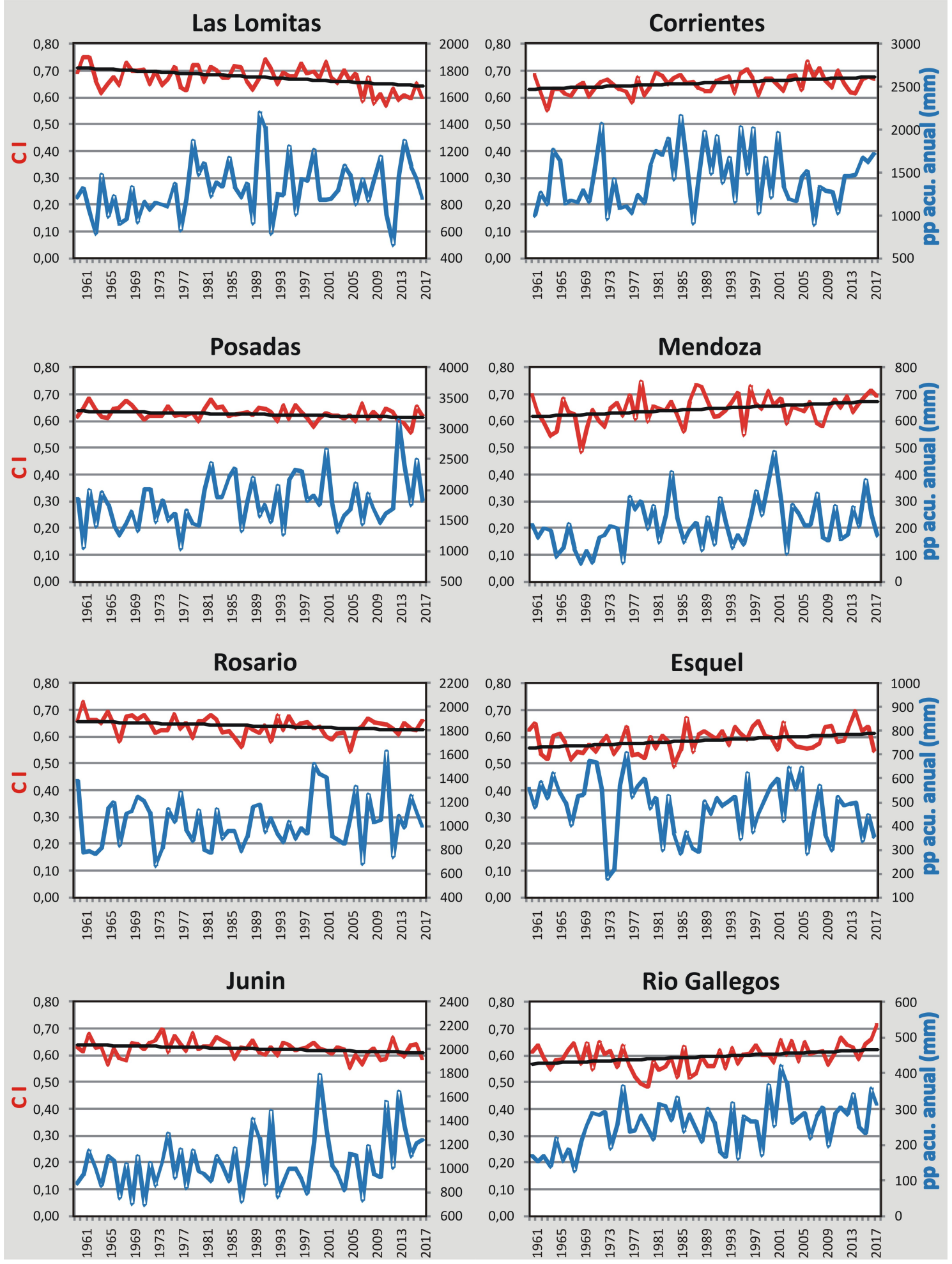

Figura 4: Valores anuales de CI 1mm (línea roja) con tendencia significativa (Mann Kendall 5\%) y en línea negra la pendiente estimada por Sen. En conjunto con los valores de precipitación acumulada anual (línea azul). 
del CI en conjunto con un aumento de la precipitación y disminución del CI y aumento de la precipitación. En el primer conjunto se encuentran Mendoza y Río Gallegos, el aumento registrado en CI significa que la precipitación está cada vez más concentrada (los días más lluviosos acumulan grandes cantidades de precipitación) y si a esto se le suma que la cantidad de precipitación también está aumentando, da por resultado que estos eventos de precipitación están concentrados y con abundante lluvia. Mientras que el otro comportamiento se observa en Las Lomitas, Posadas y Junín, en ellas el CI disminuye y la precipitación aumenta. Esto estaría indicando que hay más precipitación y la misma es más dispersa, es decir que hay más días con más lluvia.

\section{CONCLUSIONES}

El índice de concentración permite analizar a la precipitación diaria teniendo en cuenta las irregularidades que la misma presenta. Este índice evalúa a las precipitaciones con tal precisión que permite llevar a cabo un análisis estadístico de la misma que ayuda a comprender los eventos de torrencialidad y, en consecuencia, evaluar los riesgos de inundación. El índice ha sido calculado en un amplio período temporal lo cual aporta confiabilidad a las metodologías aplicadas y a los resultados hallados. Los 58 años de información diaria en 24 estaciones repartidas en el territorio argentino permite conocer una faceta de la precipitación diaria que ha sido poco estudiada. El índice calculado con un intervalo de clase $(1 \mathrm{~mm})$ toma valores entre 0,58 y 0,67 , sin un patrón definido de comportamiento exhibiendo varios centros cerrados en el centro-norte del país. Estos valores del índice permiten clasificar a las estaciones en una categoría de concentración de la precipitación moderada a alta, según la bibliografía existente. $\mathrm{Al}$ aumentar los intervalos (CI $5 \mathrm{~mm}$ y CI $10 \mathrm{~mm}$ ), los valores del índice disminuyen y a nivel espacial se hace notorio un gradiente norte - sur, en donde la estación Río Gallegos alcanza el menor valor y Las
Lomitas y Ceres presentan en máximo valor a nivel país. Con los resultados de la tendencia en el índice CI 1 se puede concluir en términos generales que ha existido un cambio significativo en la concentración de la precipitación diaria en Argentina en los últimos 60 años. Presentando variaciones de hasta 0,001 por año en el índice de concentración CI $1 \mathrm{~mm}$, tanto positivas como negativas. Las estaciones que exhiben un aumento del CI en conjunto con un aumento de la precipitación acumulada requieren mayores estudios, dado el tipo de riesgo que implica tener la precipitación concentrada en eventos de mucha lluvia.

Agradecimientos: Se agradece la asistencia técnica de P. Leal, al Banco de Datos del DCAO, al Servicio Meteorológico Nacional y a los revisores por sus interesantes y valiosos comentarios.

\section{REFERENCIAS}

Benhamrouche, A. and Martin-Vide, J., 2018: Análisis espacial de los valores de los índices de Gini y de concentración con intervalos de clase 1,5 y $10 \mathrm{~mm}$ en el litoral de la cuenca del Mediterráneo occidental (1951-2010). Geo-Eco-Trop, 42, 1: 187-198.

Brooks, C. and Carruthers, N., 1953: Handbook of Statistical Methods in Meteorology. Quarterly Journal of the Royal Meteorological Society. London: Meteorological Office.

Caloiero, T., 2014: Analysis of daily rainfall concentration in New Zealand. Natural Hazards 72:389-404.

Caloiero, T., Coscarelli, R. and Gaudio, R., 2019: Spatial and temporal variability of daily precipitation concentration in the Sardinia region (Italy). International Journal of Climatology 39: 5006-5021.

Coscarelli, R. and Caloiero, T., 2012: Analysis of daily and monthly rainfall concentration in southern Italy (Calabria region). Journal of Hydrology 416-417: 145-156.

Fernandes Teixeira, N., Teixeira Nery, J. and Martin-Vide, J., 2014: Análisis de la 
concentración temporal de la precipitación diaria en el semiárido brasileño. Cambio climático y cambio global. Publicaciones de la Asociación Española de Climatología, Almería, España pp: 145- 152. ISBN: 978-84-16027-69-9.

Kendall, M., 1962: Rank correlation methods, 3rd edn. Hafner Publishing Company, New York.

Llano, M., 2018: Spatial distribution of the daily rainfall concentration index in Argentina: comparison with other countries. Theoretical and Applied Climatology 133, 997-1007.

Mann, H., 1945: Nonparametric tests against trend Econometrica. J. Econometric Soc. 13: 245-259.

Martin-Vide, J., 2004: Spatial distribution of a daily precipitation concentration index in peninsular Spain. International Journal of Climatology 24:959-971.

Meseguer-Ruiz, O., Ponce-Philimon, P., Guijarro, J. and Sarricolea, P., 2019: Spatial distribution and trends of different precipitation variability indices based on daily data in Northern Chile between 1966 and 2015. International Journal of Climatology 39: 4595-4610.

Monjo, R. and Martin-Vide, J., 2016: Daily precipitation concentration around the world according to several indices. International Journal of Climatology 36: 3828-3838.

Nesbitt, S. Cifelli, R. and Rutledge, S., 2016: Storm morphology and rainfall characteristics of TRMM precipitation features. Monthly Weather Review 134: 2702-2721.

Núñez-González, G., 2020: Comparison of the behavior of the precipitation concentration index on global and local scale. Theoretical and Applied Climatology 139, 631-638. https: //doi.org/10.1007/s00704-019-02996-5.

Pohlert, T., 2018: Non-Parametric Trend Tests and Change-Point Detection. https://cran.r-project.org/web/ packages/trend/trend.pdf

Sarricolea, P., Meseguer-Ruiz, O., Serrano-Notivoli, R., Soto, M. and
Martin-Vide, J., 2019: Trends of daily precipitation concentration in Central-Southern Chile. Atmospheric Research 215: 85-98.

Sen, P., 1968: Estimates of the regression coefficient based on Kendall's tau. J. Am. Stat. Assoc. 63 (324), 1379-1389.

Serrano-Notivoli, R., Martín-Vid, J., Saz, M., Longares, L., Beguería, S., Sarricolea, P., Meseguer-Ruiz, O. and de Luis, M., 2018: Spatio-temporal variability of daily precipitation concentration in Spain based on a high-resolution gridded data set. International Journal of Climatology 38: 518-530.

Vélez, A., Martin-Vide, J., Royé, D. and Santaella, O., 2019: Spatial analysis of daily precipitation concentration in Puerto Rico. Theoretical and Applied Climatology 136: 1347-1355.

Wang, R., Zhang, J., Guo, E., Zhao, C. and Cao, T., 2019: Spatial and temporal variations of precipitation concentration and their relationships with large-scale atmospheric circulations across Northeast China. Atmospheric Research 222: 62-73.

Zubieta, R., Saavedra, M., Silva, Y. and Giráldez, L., 2017: Spatial analysis and temporal trends of daily precipitation concentration in the Mantaro River basin: central Andes of Peru. Stoch. Environ. Res. Risk Assess. 31:1305-1318. 